\title{
Diffusion Tensor Regularization with Constraints Preservation
}

\author{
D. Tschumperlé \\ R. Deriche \\ I.N.R.I.A, Robotvis, 2004 Rte des Lucioles \\ BP 93, 06560 Sophia Antipolis, France \\ \{David.Tschumperle,Rachid.Deriche\}@sophia.inria.fr
}

\begin{abstract}
This paper deals with the problem of regularizing noisy fields of diffusion tensors, considered as symmetric and semi-positive definite $n \times n$ matrices (as for instance $2 D$ structure tensors or DT-MRI medical images). We first propose a simple anisotropic PDE-based scheme that acts directly on the matrix coefficients and preserve the semipositive constraint thanks to a specific reprojection step. The limitations of this algorithm lead us to introduce a more effective approach based on constrained spectral regularizations acting on the tensor orientations (eigenvectors) and diffusivities (eigenvalues), while explicitely taking the tensor constraints into account. The regularization of the orientation part uses orthogonal matrices diffusion PDE's and local vector alignment procedures and will be particularly developed. For the interesting $3 D$ case, a special implementation scheme designed to numerically fit the tensor constraints is also proposed. Experimental results on synthetic and real DT-MRI data sets finally illustrates the proposed tensor regularization framework.
\end{abstract}

\section{Introduction}

Several PDE based approaches have been proposed in the last decade to tackle the problem of regularizing noisy images while preserving possible discontinuities. These approaches have been proven to be very useful for image enhancement, restoration and scale space analysis. Within this context, and since the pioneering work of Perona and Malik [21], anisotropic diffusion PDE's have been widely used through different approaches and formulations to solve the problem of scalar image denoising [1, 5, 15, 14, 24, 18, 32]. Some of these methods were recently derived within the total variation and the $\Phi$-function frameworks, in order to deal with vector valued fields, consisting of color images or geometric features that derive from any other intermediate process (image gradient, optical flow). This allowed to regularize multi-valued fields while taking possible coupling between vector components into account, including color image restoration $[1,4,12,25,26,32,29]$, regu- larization of direction fields [6, 20, 27], image inpainting or interpolation [3,7], scale space analysis. A more recent and challenging multi-valued regularization problem is the one related to data known to be constrained to specific manifolds. This is an important domain that has been recently addressed for vector directions and color images in the chromaticity-brightness or HSV spaces (using the harmonic maps theory [27], the unitary norm total variation [6] or the geometric Beltrami framework [13]). Other related works deal with images of probability vectors [19], orthonormal vector sets and rotations [30], or data on arbitrary manifolds [2]. Such constrained minimization problems usually lead to sets of coupled vector PDE's where the constraints between data are expressed in the Euler-Lagrange equations.

In this article, we go one step further and study the constrained regularization of noisy diffusion tensor fields, i.e $n \times n$ symmetric semi-positive definite matrices. Important data can be represented by such images: Structure tensors or windowed second moment matrix $([32,9,17])$, Diffusion Tensor Magnetic Resonance Images (DT-MRI) [16, 22, 31] and Covariance matrices. We first propose an anisotropic scheme that acts directly on each matrix coefficient while preserving the symmetry and semi-positivity constraints of the tensors, thanks to a simple reprojection step (section 3). The performance and limitations of this method lead us to propose a more effective method (section 4), based on a spectral decomposition of the tensors, followed by a constrained regularization of the spectral elements : the tensor orientations and diffusivities. This approach, whose key idea is the use of a new orthogonal matrix diffusion technique (section 4.3) coupled with a local direction vector alignment (section 4.4), constructs a complete and coherent regularization process, avoiding any reprojection to the tensor space or any reconstruction of the final tensors from partially regularized data.

Finally, a numerical scheme specially designed for 3D tensor regularization purposes is proposed (section 5) and illustrated with the regularization of noisy synthetic and real DT-MRI data sets (section 6). 


\section{Context and Notations}

In this paper, a diffusion tensor $\mathbf{T}=\left(T_{i, j}\right)$ is considered as a symmetric and semi-positive definite $n \times n$ matrix :

$$
T_{i, j}=T_{j, i} \quad \text { and } \quad \forall \mathbf{x} \in \mathbb{R}^{n},(\mathbf{T} \mathbf{x} \cdot \mathbf{x}) \geq 0
$$

$\mathbf{T}$ can be expressed with its eigenvalues $\lambda_{l}$ and its corresponding eigenvectors $\mathbf{u}^{[l]}=\left(u_{i}^{[l]}\right)$. Its spectral decomposition is :

$$
\mathbf{T}=\sum_{l=1}^{n} \lambda_{l} \mathbf{u}^{[l]} \mathbf{u}^{[l]}=\mathbf{U} \mathbf{D} \mathbf{U}^{T}
$$

where $\mathbf{U}=\left(\mathbf{u}^{[1]}|\ldots| \mathbf{u}^{[n]}\right)$ is the $n \times n$ orthogonal matrix of the unit eigenvectors columns $\mathbf{u}^{[l]}$, forming an orthonormal vector basis, while $\mathbf{D}=\operatorname{diag}\left(\lambda_{1}, \ldots, \lambda_{n}\right)$ is the corresponding diagonal matrix of the positive eigenvectors, supposed ordered : $\lambda_{1} \geq \lambda_{2} \geq \cdots \geq \lambda_{n} \geq 0$.

The spectral decomposition separates the orientation feature $\mathbf{U}$ and the diffusivity feature $\mathbf{D}$ of a tensor $\mathbf{T}$.

For the 3D case ( $n=3$ ), a natural representation of $\mathbf{T}$ is then an ellipsoid whose axes and radiuses are respectively given by $\mathbf{u}^{[1]}, \mathbf{u}^{[2]}, \mathbf{u}^{[3]}$ and $\lambda_{1}, \lambda_{2}, \lambda_{3}$.

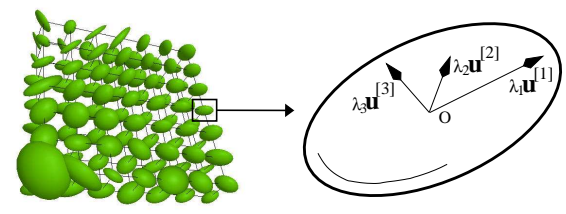

Figure 1. View of a 3D diffusion tensor field $\mathcal{T}$.

We are interested in regularizing noisy diffusion tensor fields $\mathcal{T}$ defined on a continuous domain $\Omega$ of $\mathbb{R}^{p}$ (usually $p=1,2,3)$. This work is motivated by the restoration of 3D DT-MRI medical images of the brain (as described in $[16,22]$ ), but can be used without restriction to general symmetric and semi-positive definite $n \times n$ matrices.

\section{A PDE regularization scheme acting on the tensor coefficients}

Image regularization with PDE is often based on the minimization of a functional expressed with a measure of the local data variation. Minimizing the low variations while preserving the high ones (considered as important discontinuities) leads to anisotropic diffusion PDE's via EulerLagrange equations, which regularize the image. In our case, a tensor field $\mathcal{T}$ could be seen as a multi-valued image where each point of the field has $n^{2}$ components. Then, a natural idea would be to evolve the tensor components $T_{i, j}$ with classic vector diffusion PDE's, as for instance :

$$
\frac{\partial T_{i, j}}{\partial t}=\alpha\left(T_{0_{i, j}}-T_{i, j}\right)+\operatorname{div}\left(\frac{\Phi^{\prime}(\|\nabla \mathcal{T}\|)}{\|\nabla \mathcal{T}\|} \nabla T_{i, j}\right)
$$

coming from the minimization of

$$
E(\mathcal{T})=\int_{\Omega}\left[\frac{\alpha}{2}\left(\mathcal{T}-\mathcal{T}_{0}\right)^{2}+\Phi(\|\nabla \mathcal{T}\|)\right] d \Omega
$$

where $\|\nabla \mathcal{T}\|=\sqrt{\sum_{k, l}\left\|\nabla T_{k, l}\right\|^{2}}$ is a measure of the local tensor variation, $\mathcal{T}_{0}$ is the initial (noisy) matrix field, and $\Phi$ is a regularization function (see $[8,10,15,21,23,28]$ for the scalar case). Note how the term $\|\nabla \mathcal{T}\|$ inside the divergence operator acts as a coupling term between all the matrix coefficients. It is also easy to see that the PDE (2) intrinsically preserves the matrix symmetry and can then be applied only on the upper triangular part of T (i.e $n(n+1) / 2$ components instead of $n^{2}$ ).

As expected, the semi-positive definite constraint needs more attention. One can re-project at each iteration the regularized matrices $\mathbf{T}$ into the semi-positive space, with respect to the Frobenius norm (see the appendix) :

$\mathcal{P}(\mathbf{T})=\mathbf{U} \operatorname{diag}\left(\tilde{\lambda_{1}}, \ldots, \tilde{\lambda_{n}}\right) \mathbf{U}^{T} \quad / \begin{cases}\tilde{\lambda}_{l}=\lambda_{l} & \left(\text { if } \lambda_{l} \geq 0\right) \\ \tilde{\lambda_{l}}=0 & \left(\text { if } \lambda_{l}<0\right)\end{cases}$

This reprojection step ensures the semi-positive property of the tensors during the PDE flow. However, this technique has some drawbacks :

- The reprojection requires a spectral decomposition of the matrix field (to get the $\lambda_{l}$ ), at each PDE iteration which is a very time consuming procedure.

- We do not have a direct control on the spectral elements of the tensors, which are primordial : They decompose the tensor informations (and so the noise) and are relevant informations to drive the regularization process. One consequence of a direct matrix diffusion with eq.(2) is the eigenvalue smoothing effect that happens for neighbouring tensors with near orthogonal directions (Fig.2a,b,c, see how the size of the ellipsoids are not preserved, in Fig.2c).

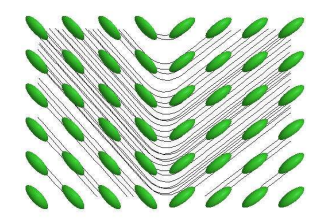

a) Synthetic $3 \mathrm{D}$ tensor field $\boldsymbol{\mathcal { T }}$

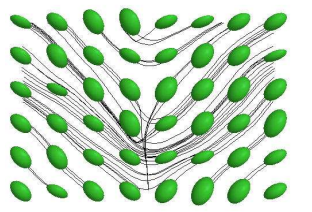

c) Direct matrix diffusion (eq.(2))

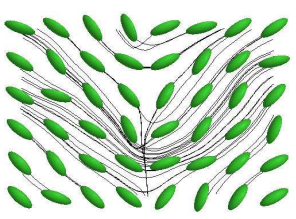

b) With noisy orientations

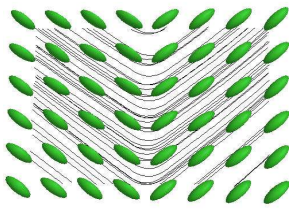

d) Spectral regularization (eq.(7))
Figure 2. Synthetic tensor field regularization. 


\section{A spectral regularization approach}

More generally, we rather propose to work directly on the spectral decomposition of the noisy tensor field while preserving the tensor constraints in the spectral space (Fig.2d).

\subsection{Motivations and constraints}

The spectral elements provide significant structural informations, that could be used to regularize a tensor field :

- For DT-MRI images, D measures the water molecule velocity in the brain fibers, while the eigenvectors $\mathbf{u}^{[l]}$ provide important clues to the structure and geometric organization of these fibers. Significant values can then be computed [16] : mean diffusivity : $\operatorname{Tr}=\lambda_{1}+\lambda_{2}+\lambda_{3}$, partial anisotropy : $F A=\sqrt{\frac{\left(\lambda_{1}-\lambda_{2}\right)^{2}+\left(\lambda_{1}-\lambda_{3}\right)^{2}+\left(\lambda_{2}-\lambda_{3}\right)^{2}}{2\left(\lambda_{1}^{2}+\lambda_{2}^{2}+\lambda_{3}^{2}\right.}}$, volume ratio $: V R=27 \lambda_{1} \lambda_{2} \lambda_{3} /\left(\lambda_{1}+\lambda_{2}+\lambda_{3}\right)$.

- For structure tensors of color images [32], D and U measure the color variations and their corresponding directions. - For covariance matrices, D represents the standard deviations of the data dispersion along the main axes $\mathbf{U}$.

These few examples clearly illustrates how well the spectral decomposition of a tensor is directly related to a better understanding of its structure. Moreover, the tensor constraints (1) are easily expressed in the spectral domain :

$$
\left\{\begin{array}{lll}
\text { Semi-positivity : } & \forall l & \lambda_{l} \geq 0 \\
\text { Orthogonality : } & \forall k, l, & \mathbf{u}^{[k]} \cdot \mathbf{u}^{[l]}=\delta_{k, l}
\end{array}\right.
$$

D ensures the tensor semi-positivity while the orthogonality of $\mathbf{U}$ ensures the symmetry of the corresponding tensor. Then, our spectral method will be based on two constrained and coupled regularizations acting on $\mathbf{D}$ and $\mathbf{U}$.

\subsection{Regularization of the tensor diffusivities}

Different anisotropic PDE's can be used to regularize the tensor diffusivities $\mathbf{D}=\operatorname{diag}\left(\lambda_{l}\right)$, depending on the considered application. For instance,the following diffusion schemes could be considered for analysis :

- Process each eigenvalue $\lambda_{l}$ separately:

$$
\frac{\partial \lambda_{l}}{\partial t}=\alpha\left(\lambda_{0_{l}}-\lambda_{l}\right)+\operatorname{div}\left(\frac{\Phi^{\prime}\left(\left\|\nabla \lambda_{l}\right\|\right)}{\left\|\nabla \lambda_{l}\right\|} \nabla \lambda_{l}\right)
$$

- Process the vector $\boldsymbol{\lambda}=\left(\lambda_{l}\right)$ using vector diffusion PDE's :

$$
\frac{\partial \lambda_{l}}{\partial t}=\alpha\left(\lambda_{0_{l}}-\lambda_{l}\right)+\operatorname{div}\left(\frac{\Phi^{\prime}(\|\nabla \boldsymbol{\lambda}\|)}{\|\nabla \boldsymbol{\lambda}\|} \nabla \lambda_{l}\right)
$$

- Include a-priori spectral informations inside the divergence operator, in order to drive the diffusion process :

$$
\frac{\partial \lambda_{l}}{\partial t}=\alpha\left(\lambda_{0_{l}}-\lambda_{l}\right)+\operatorname{div}\left(\frac{\Phi^{\prime}(\|\nabla \lambda\|, F A, V R, \ldots)}{\|\nabla \lambda\|} \nabla \lambda_{l}\right)
$$

Working on the spectral domain allows much more freedom in the choice of the diffusion terms, than a direct matrix restoration. The semi-positivity constraint is imposed by using a discretized scheme that satisfies the maximum and minimum principle [1], then the tensor eigenvalues will always be positive. Fig.3 illustrates the regularization of eigenvalues from a DT-MRI image of the brain.

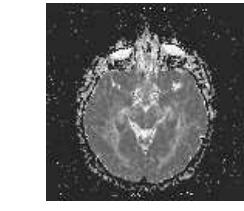

a) Brain DT-MRI diffusivities

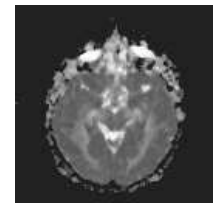

b) Regularized diffusivities
Figure 3. Eigenvalues regularization

\subsection{Tensor orientation regularization}

The difficult part of our spectral regularization method comes from the preservation of the orthogonality of $\mathbf{U}$ during the PDE flow. Indeed, using unconstrained vector diffusion PDE's, or norm constrained PDE's (as in $[6,20,27,29]$ ) on each eigenvector $\mathbf{u}^{[l]}$ (the columns of $\mathbf{U}$ ) doesn't preserve intrinsically the orthonormal constraints (4). Note how the unitary norm is well preserved on Fig.4c, but not the orthogonal angles.

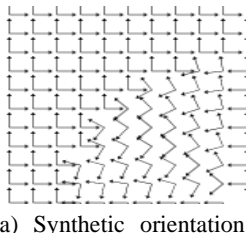

a) Synthetic orientation field $\mathbf{U}$

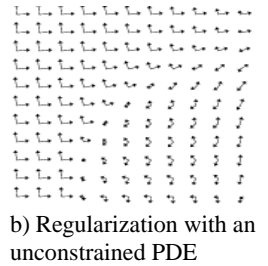
unconstrained PDE

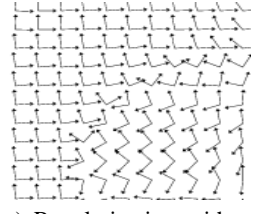

c) Regularization with a norm constrained PDE

\section{Figure 4. Classic PDE's fail to regularize U}

Following the idea of our previous regularization work acting on orthonormal vector sets [30], we propose to minimize this general functional with respect to $\mathbf{u}^{[l]}$,

$$
E(\mathbf{U})=\int_{\Omega} \Psi\left(\mathbf{u}^{[1]}, . ., \mathbf{u}^{[n]},\left\|\nabla \mathbf{u}^{[1]}\right\|, \ldots,\left\|\nabla \mathbf{u}^{[n]}\right\|\right) d \Omega
$$

while preserving the spectral orthonormal constraints (4). $\Psi$ is a free regularization functional (depending on the diffusion behaviour we desire), as for instance the one based on the $\Phi$-function formulation (3) :

$$
\Psi(.)=\sum_{l=1}^{n} \frac{\alpha}{2}\left(\mathbf{u}^{[l]}-\mathbf{u}_{0}^{[l]}\right)^{2}+\Phi\left(\left\|\nabla \mathbf{u}^{[l]}\right\|\right)
$$

The orthonormal constraints are introduced in the minimization functional thanks to $n^{2}$ Lagrange multipliers $\lambda_{p q}$, leading to the unconstrained minimization of

$$
E^{*}(\mathbf{U}, \lambda)=E(\mathbf{U})+\int_{\Omega} \sum_{(p, q) \in[1 \ldots n]} \lambda_{p q}\left(\mathbf{u}^{[p]} \cdot \mathbf{u}^{[q]}-\delta_{p q}\right) d \Omega
$$

with respect to $\mathbf{u}^{[l]}$ and $\lambda_{p q}$. Note that, as the dot product and $\delta_{p q}$ are symmetric, $\lambda_{p q}$ and $\lambda_{q p}$ are equal. developing 
the Euler-Lagrange equations and solving them with respect to $\lambda_{p q}$ gives the following set of coupled and constrained PDE's acting on the column vectors $\mathbf{u}^{[l]}$ of $\mathbf{U}$ and preserving its orthonormal properties (see [30]) :

$$
\frac{\partial \mathbf{u}^{[k]}}{\partial t}=\sum_{l=1}^{n}\left(\mathcal{L}(E)^{[l]} \cdot \mathbf{u}^{[k]}\right) \mathbf{u}^{[l]}-\mathcal{L}(E)^{[k]}
$$

and if we consider the function $\Psi$ of eq.(5), we have :

$$
\mathcal{L}(E)_{i}^{[k]}=\alpha\left(u_{i}^{[k]}-u_{i_{0}}^{[k]}\right)-\operatorname{div}\left(\frac{\Phi^{\prime}\left(\left\|\nabla \mathbf{u}^{[k]}\right\|\right)}{\left\|\nabla \mathbf{u}^{[k]}\right\|} \nabla u_{i}^{[k]}\right)
$$

More generally, $\mathcal{L}(E)^{[k]} \in \mathbb{R}^{n}$ is the Lagrangian vector of the unconstrained energy $E(\mathbf{U})$ subject to the vector $\mathbf{u}^{[k]}$. The beauty of this formulation is the independence between the unconstrained Lagrangian and the orthonormal constrained terms. The choice of the regularization functional is then fully open. Fig.5a,b,c shows the application of such orthonormal constrained PDE's on a synthetic 3D orientation field (the three unit and orthogonal eigenvectors $\mathbf{u}^{[l]}$ are represented). Note how the discontinuities are well preserved by the anisotropic behaviour of the diffusion.

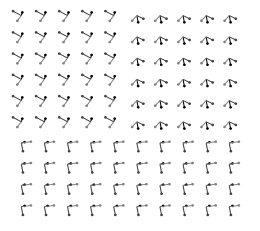

a) Synthetic tensor orientation field $\mathbf{U}$

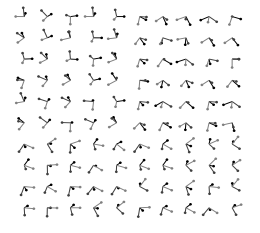

b) With orientation noise $\left(\sigma=30^{\circ}\right)$

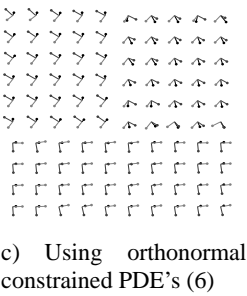

Figure 5. Orthonormal constrained PDE's

This framework can also be used to restore other orientation features, like fields of rotation matrices, direction vectors, color chromaticity ([30]).

\subsection{A local alignment method}

When dealing with diffusion tensors, one has to take care of the non-unicity of the spectral decomposition $\mathbf{T}=\sum_{k=1}^{n} \lambda_{k} \mathbf{u}^{[k]} \mathbf{u}^{[k]^{T}}$. Flipping one eigenvector direction while keeping its orientation (i.e considering $-\mathbf{u}^{[l]}$ instead of $\mathbf{u}^{[l]}$ ) gives the same tensor $\mathbf{T}: 2^{n}$ configurations can represent its orientation $\mathbf{U}$. This means that a constant tensor field may be decomposed into highly discontinuous eigenvector fields, disturbing the anisotropic regularization process with false discontinuity detections. This non-unicity problem happens also with other orientation representations, such as quaternions, Euler angles, or rotation vectors. To overcome this problem, a local eigenvector alignment process can be made before applying the PDE on each tensor of the field $\mathcal{T}$. The idea is to align the neighbouring eigenvector directions with the current one. This is done by minimizing the angles between them, constraining the dot product to be positive by flipping the neighbouring eigenvectors if necessary :

$\forall N \in \mathcal{V}(M), \quad \mathbf{u}^{\tilde{[}]}(N)=\operatorname{sign}\left(\mathbf{u}^{[i]}(N) \cdot \mathbf{u}^{[i]}(M)\right) \mathbf{u}^{[i]}(N)$ where $\mathcal{V}(M)$ is a neighbourhood of $M$ (Fig.6). This local operation allows to act on the vector orientations while ignoring the direction information. Then, we can apply the orthogonal constrained diffusion PDE eq.(6). The importance of this procedure is shown on Fig.9c, for regularization of real DT-MRI fields.

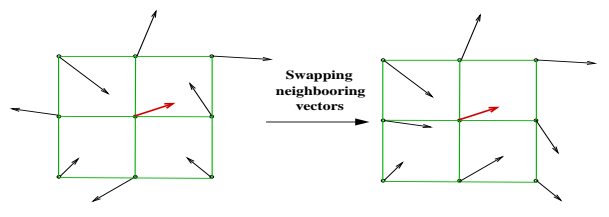

Figure 6. Local vector alignment procedure

\section{3D implementation issues}

Numerical schemes using finite differences are not well adapted for PDE's acting on data constrained on curved manifolds. Fig.7 illustrates the problem for a vector constrained to a sphere : The time step has to be very small, in order to numerically stay on the manifold.

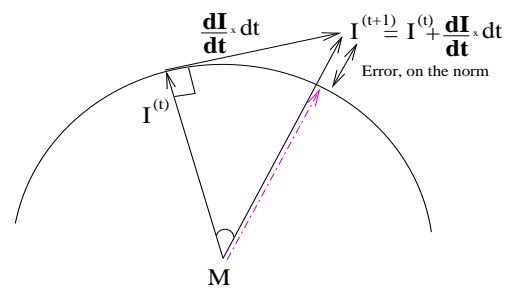

\section{Figure 7. Finite difference schemes and curved manifolds.}

Let us rewrite the orientation diffusion PDE's (6) for 3D tensors. For simplicity reasons, we will write : $\mathbf{u}^{[1]}=\mathbf{I}$, $\mathbf{u}^{[2]}=\mathbf{J}$ and $\mathbf{u}^{[3]}=\mathbf{K}$ :

$$
\left\{\begin{array}{l}
\frac{\partial \mathbf{I}}{\partial t}=\mathcal{L}^{\mathbf{I}}-\left(\mathcal{L}^{\mathbf{I}} . \mathbf{I}\right) \mathbf{I}-\left(\mathcal{L}^{\mathbf{J}} . \mathbf{I}\right) \mathbf{J}-\left(\mathcal{L}^{\mathbf{K}} . \mathbf{I}\right) \mathbf{K} \\
\frac{\partial \mathbf{J}}{\partial t}=\mathcal{L}^{\mathbf{J}}-\left(\mathcal{L}^{\mathbf{I}} . \mathbf{J}\right) \mathbf{I}-\left(\mathcal{L}^{\mathbf{J}} . \mathbf{J}\right) \mathbf{J}-\left(\mathcal{L}^{\mathbf{K}} . \mathbf{J}\right) \mathbf{K} \\
\frac{\partial \mathbf{K}}{\partial t}=\mathcal{L}^{\mathbf{K}}-\left(\mathcal{L}^{\mathbf{I}} . \mathbf{K}\right) \mathbf{I}-\left(\mathcal{L}^{\mathbf{J}} . \mathbf{K}\right) \mathbf{J}-\left(\mathcal{L}^{\mathbf{K}} . \mathbf{K}\right) \mathbf{K}
\end{array}\right.
$$

where $\mathcal{L}^{\mathbf{u}}$ is the Lagrangian vector given in eq.(6). These equations can be factorized, using the double cross product formula $\mathbf{u} \times(\mathbf{v} \times \mathbf{w})=(\mathbf{u} . \mathbf{w}) \mathbf{v}-(\mathbf{u . v}) \mathbf{w}$ :

$$
\frac{\partial \mathbf{I}}{\partial t}=\omega \times \mathbf{I}, \quad \frac{\partial \mathbf{J}}{\partial t}=\omega \times \mathbf{J}, \quad \text { and } \quad \frac{\partial \mathbf{K}}{\partial t}=\omega \times \mathbf{K}
$$

with $\omega=\mathbf{I} \times \mathcal{L}^{\mathbf{I}}+\mathbf{J} \times \mathcal{L}^{\mathbf{J}}+\mathbf{K} \times \mathcal{L}^{\mathbf{K}}$. This physically means that we apply the same infinitesimal 3D rotation vector $\omega d t$ in order to compute the displacements $d \mathbf{I}, d \mathbf{J}$ and $d \mathbf{K}$ of the eigenvectors. In this case, a natural scheme is : 
- Compute the vector $\omega=\mathbf{I} \times \mathcal{L}^{\mathbf{I}}+\mathbf{J} \times \mathcal{L}^{\mathbf{J}}+\mathbf{K} \times \mathcal{L}^{\mathbf{K}}$. - Apply the same infinitesimal rotation $\omega d t$ to $\mathbf{I}, \mathbf{J}$ and $\mathbf{K}$ : $\mathbf{I}_{(t+1)}=\mathcal{R}_{\omega d t}\left(\mathbf{I}_{(t)}\right) \quad$ (using quaternions for instance). The orthonormal constraints are numerically preserved, since the error due to $(d t \neq 0)$ only affects the rotation parameters, which are common for all eigenvectors $\mathbf{u}^{[l]}$.

\section{Applications}

- We first applied our proposed framework, in order to compare the behaviour of the two equations (2) and (6) on a synthetic 3D tensor field (Fig.8). The noisy image is obtained by adding uniform noise on the tensor orientations and diffusivities. Note how the eigenvalues are smoothed with a direct matrix regularization approach eq.(2), while the spectral regularization allows more control on the tensor diffusion (eq.(6)).

- The main application of interest that we considered is the restoration of DT-MRI images of the brain. The data were kindly provided by the SHFJ-CEA, thanks to J.F Mangin. Computing fiber tracking on regularized images allows to get more coherent bunch of fibers in the white matter. the main streamlines are computed (following at each point the main eigenvector $\mathbf{u}^{[1]}$ ) and displayed with black lines, for the original data (Fig.9a), the regularized data with the direct approach (Fig.9b), and the regularized data with the spectral method (Fig.9c,d). Notice that the local alignment process is essential in the global regularization process.

\section{Conclusions \& Perspectives}

In this paper, we proposed and compared two different methods for regularizing $n \times n$ diffusion tensor fields, while preserving the symmetry and semi-positive definite constraints of the matrices. We first showed the difficulties and limitations that a direct matrix regularization approach causes. Then a spectral regularization method has been proposed, acting on the whole spectral components of the tensors and avoiding any matrix reprojection. We proposed a solution to solve the main difficulty of this approach : the regularization of the tensor orientations, thanks to orthonormal constrained diffusions and local vector alignments. For the 3D case, we proposed an efficient numerical scheme that follows the constraints, as well as we applied it to restore DT-MRI images.

\section{Appendix : The reprojection method}

Let $\mathbf{A}=\mathbf{U D U}^{T}$ be a symmetric matrix ( $\mathbf{U}$ is orthogonal and $\mathbf{D}=\operatorname{diag}\left(\lambda_{l}\right)$ ). We are looking for the semi-positive definite matrix $\mathbf{B}$ that minimize the Frobenius norm :

$$
\|\mathbf{A}-\mathbf{B}\|_{F}^{2}=\left\|\mathbf{U} \mathbf{D} \mathbf{U}^{T}-\mathbf{B}\right\|_{F}^{2}=\left\|\mathbf{D}-\mathbf{U}^{T} \mathbf{B} \mathbf{U}\right\|_{F}^{2}
$$

(the norm is independent of the basis). $\mathbf{U}^{T} \mathbf{B U}$ is symmetric. Let us call $\mathbf{D}^{\prime}$ the matrix of its diagonal elements and $\mathbf{S}$ its lower triangular matrix such as : $\mathbf{U}^{T} \mathbf{B U}=\mathbf{D}^{\prime}+\mathbf{S}+\mathbf{S}^{T}$.
Then, we have : $\|\mathbf{A}-\mathbf{B}\|_{F}^{2}=\left\|\mathbf{D}-\mathbf{D}^{\prime}\right\|_{F}^{2}+2\|\mathbf{S}\|_{F}^{2}$. The minimum is reached when $\mathbf{S}=0$, i.e $\mathbf{B}=\mathbf{U D}^{\prime} \mathbf{U}^{T}$, and then $\|\mathbf{A}-\mathbf{B}\|_{F}^{2}=\left\|\mathbf{D}-\mathbf{D}^{\prime}\right\|_{F}^{2}=\sum_{i=1}^{n}\left(\lambda_{l}-\mu_{i}\right)^{2}$, where $\mu_{1}, \ldots, \mu_{n}$ are the positive eigenvalues of $\mathbf{B}$.

$$
\|\mathbf{A}-\mathbf{B}\|_{F}^{2} \text { is minimum } \Longleftrightarrow \begin{cases}\mu_{i}=\lambda_{l} & \text { (if } \left.\lambda_{l} \geq 0\right) \\ \mu_{i}=0 & \text { (if } \left.\lambda_{l}<0\right)\end{cases}
$$

\section{Acknowledgements}

The authors would like to thank J.F Mangin (SHFJ-CEA), O.Faugeras and T. Papadopoulo (Robotvis/INRIA).

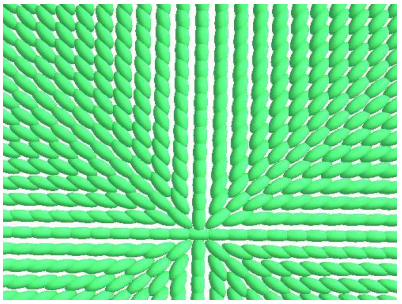

a) Synthetic $3 \mathrm{D}$ tensor field

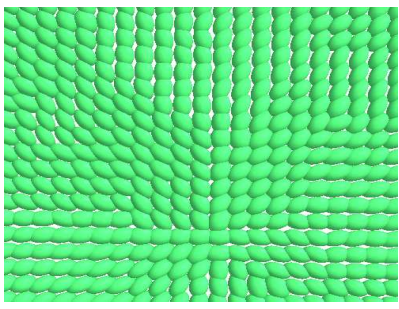

c) After direct matrix restoration eq.(2).

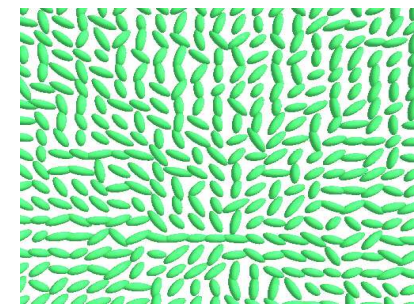

b) Adding random noise.

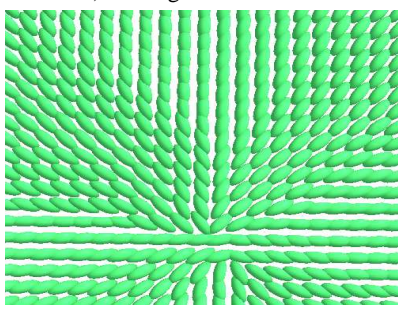

d) After spectral restoration eq.(6).

\section{Figure 8. Synthetic and noisy 3D tensor fields restoration.}

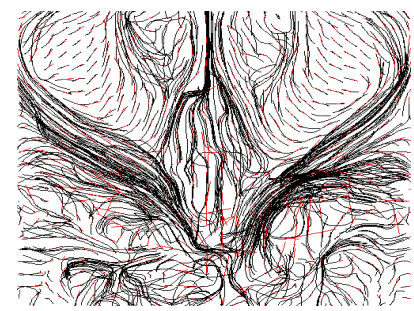

a) Streamlines of a real DT-MRI field.

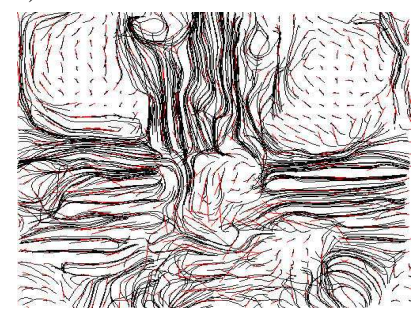

c) Spectral restoration eq.(6) without local alignment.

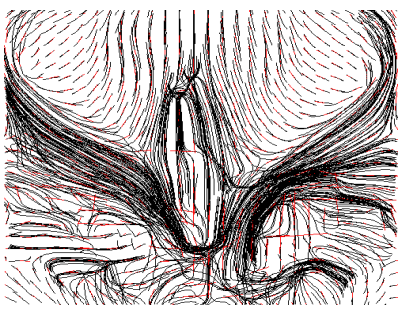

b) Direct matrix restoration eq.(2).

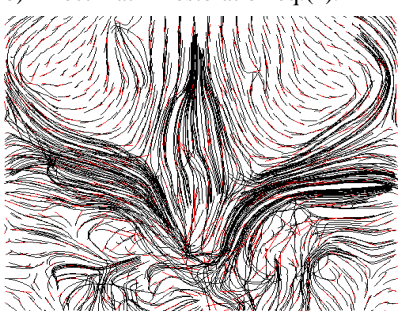

d) Spectral restoration eq.(6) with local alignment.
Figure 9. Streamlines computation on a real DT-MRI data set. 


\section{References}

[1] Bart M. ter Haar Romeny. Geometry-driven diffusion in computer vision. Computational imaging and vision. Kluwer Academic Publishers, 1994.

[2] M. Bertalmio, L.-T. Cheng, S. Osher, and G. Sapiro. Variational problems and partial differential equations on implicit surfaces: The framework and examples in image processing and pattern formation. UCLA Research Report, June 2000.

[3] M. Bertalmio, G. Sapiro, V. Caselles, and C. Ballester. Image inpainting. In K. Akeley, editor, Proceedings of the SIGGRAPH, pages 417-424. ACM Press, ACM SIGGRAPH, Addison Wesley Longman, 2000.

[4] P. Blomgren and T. Chan. Color tv: Total variation methods for restoration of vector-valued images. IEEE Trans. Imag. Proc., 7(3):304-309, 1998. Special Issue on Partial Differential Equations and Geometry-Driven Diffusion in Image Processing and Analysis.

[5] V. Caselles, J. Morel, G. Sapiro, and A. Tannenbaum. Introduction to the special issue on partial differential equations and geometry-driven diffusion in image processing and analysis. IEEE Transactions on Image Processing, 7(3):269273, 1998.

[6] T. Chan and J. Shen. Variational restoration of non-flat image features : Models and algorithms. Research Report. Computational and applied mathematics department of mathematics Los Angeles., June 1999.

[7] T. F. Chan and J. Shen. Non-texture inpaintings by curvature-driven diffusions (cdd). Technical Report 00-35, Department of Mathematics, UCLA, Los Angeles, Sept. 2000.

[8] P. Charbonnier, G. Aubert, M. Blanc-Féraud, and M. Barlaud. Two deterministic half-quadratic regularization algorithms for computed imaging. In Proceedings of the International Conference on Image Processing, volume II, pages 168-172, 1994.

[9] M. A. Förstner and E. Gülch. A fast operator for detection and precise location of distinct points, corners and centers of circular features. In Proceedings of the Intercommission Workshop of the International Society for Photogrammetry and Remote Sensing, Interlaken, Switzerland, 1987.

[10] S. Geman and D. McClure. Bayesian image analysis : an application to single photon emission tomography. Amer. Statist. Assoc., pages 12-18, 1985.

[11] G. H. Granlund and H. Knutsson. Signal Processing for Computer Vision. Kluwer Academic Publishers, 1995.

[12] R. Kimmel, R. Malladi, and N. Sochen. Images as embedded maps and minimal surfaces: movies, color, texture, and volumetric medical images. International Journal of Computer Vision, 39(2):111-129, Sept. 2000.

[13] R. Kimmel and N. Sochen. Orientation diffusion or how to comb a porcupine. Technical Report 2000-02, CIS, 2000. Accepted to special issue on PDEs in Image Processing, Computer Vision, and Computer Graphics, Journal of Visual Communication and Image Representation, 2000.

[14] P. Kornprobst, R. Deriche, and G. Aubert. Image coupling, restoration and enhancement via PDE's. In Proceedings of the International Conference on Image Processing, volume 4, pages 458-461, Santa Barbara, California, Oct. 1997.

[15] P. Kornprobst, R. Deriche, and G. Aubert. Nonlinear operators in image restoration. In Proceedings of the International Conference on Computer Vision and Pattern Recognition, pages 325-331, Puerto Rico, June 1997. IEEE Computer Society, IEEE.

[16] D. Le Bihan. Methods and applications of diffusion mri. In I. Young, editor, Magnetic Resonance Imaging and Spectroscopy in Medicine and Biology. John Wiley and Sons, 2000.

[17] T. Lindeberg. Scale-Space Theory in Computer Vision. Kluwer Academic Publishers, 1994.

[18] J. Morel and S. Solimini. Segmentation of images by variational methods: A constructive approach. Rev. Math. Univ. Complut. Madrid, 1:169-182, 1988.

[19] A. Pardo and G. Sapiro. Vector probability diffusion. International Conference on Image Processing, Sept. 2000.

[20] P. Perona. Orientation diffusions. IEEE Transactions on Image Processing, 7(3):457-467, Mar. 1998.

[21] P. Perona and J. Malik. Scale-space and edge detection using anisotropic diffusion. IEEE Transactions on Pattern Analysis and Machine Intelligence, 12(7):629-639, July 1990.

[22] C. Poupon. Détection des faisceaux de fibres de la substance blanche pour l'étude de la connectivité anatomique cérébrale. $\mathrm{PhD}$ thesis, Ecole Nationale Supérieure des Télécommunications, Dec. 1999.

[23] L. Rudin, S. Osher, and E. Fatemi. Nonlinear total variation based noise removal algorithms. Physica D, 60:259-268, 1992.

[24] G. Sapiro. Geometric Partial Differential Equations and Image Analysis. Cambridge University Press, 2001.

[25] G. Sapiro and D. Ringach. Anisotropic diffusion of multivalued images with applications to color filtering. IEEE Transactions on Image Processing, 5(11):1582-1585, 1996.

[26] N. Sochen, R. Kimmel, and R. Malladi. From high energy physics to low level vision. Technical Report 39243, LBNL report, UC Berkeley, 1996.

[27] B. Tang, G. Sapiro, and V. Caselles. Diffusion of general data on non-flat manifolds via harmonic maps theory: The direction diffusion case. The International Journal of Computer Vision, 36(2):149-161, Feb. 2000.

[28] A. Tikhonov. Regularization of incorrectly posed problems. Soviet. Math. Dokl., 4:1624-1627, 1963.

[29] D. Tschumperlé and R. Deriche. Constrained and unconstrained pdes for vector image restoration. 12th Scandinavian Conference on Image Analysis, pages 153-160, June 2001.

[30] D. Tschumperle and R. Deriche. Regularization of orthonormal vector sets using coupled PDE's. Proceedings of IEEE Workshop on Variational and Level Set Methods in Computer Vision, pages 3-10, July 2001.

[31] B. Vemuri, Y. Chen, M. Rao, T. McGraw, T. Mareci, and Z. Wang. Fiber tract mapping from diffusion tensor mri. In 1st IEEE Workshop on Variational and Level Set Methods in Computer Vision (VLSM'01), July 2001.

[32] J. Weickert. A review of nonlinear diffusion filtering. ScaleSpace Theory in Computer Vision, Lecture Notes in Comp. Science (Springer, Berlin), 1252:3-28, 1997. Invited Paper. 